\title{
७MONÇÕES
}

\section{Le Brésil dans les négociations sur la biodiversité au-delà des juridictions nationales}

\author{
O Brasil nas negociações sobre a biodiversidade além das \\ jurisdições nacionais
}

\author{
Brazil in biodiversity negotiations beyond national \\ jurisdictions
}

\author{
Igor Magri de Queiroz \\ Instituto de Relações Internacionais - IREL, Universidade de Brasília - UnB \\ Brasília, DF, Brasil \\ E-mail: igormagriq@gmail.com \\ OrclD: https://orcid.org/0000-0002-1214-6934
}

Resumé: Les négociations pour un instrument contraignant pour la biodiversité au-delà des zones de juridiction nationale (BADJN) sont en cours sous l'égide des Nations Unies. Ce processus est le résultat de plus de 15 ans de discussions internationales, qui divise les pays encore une fois sur comment résoudre les écarts de la réglementation de la BADJN. Dans cette étude, basée sur une recherche de sources primaires et secondaires, nous cartographions la position d'un pays qui a une grande responsabilité par rapport au développement durable, le Brésil. Les résultats de notre recherche montrent que ce pays continue à consacrer peu de ressources institutionnelles à la question, bien qu'elle puisse être utilisée pour son développement durable. Cela conduit à deux constatations : un manque de diplomatie stratégique pour la question et une concentration des ressources pour les questions océaniques sur sa juridiction nationale. Pourtant, le pays continue à défendre ses positions historiques, principalement à travers sa participation au groupe de pays dans la négociation, même avec la transition des gouvernements, bien que plus faible plus récemment.

Mots-clés: Biodiversité au-delà des zones de juridiction nationale ; Brésil; Océans. 
Resumo: As negociações para um acordo vinculante para a biodiversidade além das jurisdições nacionais (BAJN) estão em andamento sob as Nações Unidas. Este processo é o resultado de mais de 15 anos de discussões internacionais que dividem os países sobre como preencher as lacunas dos regulamentos da BAJN. Neste estudo, com base em uma pesquisa de fontes primárias e secundárias, mapeamos a posição de um país com grande responsabilidade pelo desenvolvimento sustentável o Brasil. Os resultados da pesquisa mostram que este país continua dedicando poucos recursos institucionais ao tema, embora este pudesse ser utilizado para fomentar o seu próprio desenvolvimento sustentável. Isto leva a duas constatações: uma falta de diplomacia estratégica para o tema e uma concentração de recursos para questões oceânicas que estejam sobre sua jurisdição nacional. Entretanto, o país continua a defender suas posições históricas, principalmente por meio de sua participação em grupos de países na negociação, mantendo-a mesmo com a transição de governos, embora com menos força mais recentemente.

Palavras-chave: Biodiversidade além das jurisdições nacionais; Brasil; Oceanos.

Abstract: Negotiations for a binding instrument for marine biodiversity of areas beyond national jurisdiction (BBNJ) are underway under the United Nations. This process is the result of more than 15 years of international discussions, which divides countries over how to fill the gaps in BBNJ regulations. In this study, based on a research of primary and secondary sources, we map the position of a country with great responsibility for sustainable development, Brazil. The results of our research show that this country continues to devote few institutional resources to the issue, although it could be used for its national sustainable development, which points to a lack of strategic diplomacy for the issue and a concentration of resources for ocean issues on its national jurisdiction. Nevertheless, the country continues to defend its historical positions, mainly through its participation in groups of countries in the negotiation, even with the transition of governments, although weaker more recently.

Keywords: Biodiversity beyond national jurisdictions; Brazil; Oceans.

Recebido em

21/02/2020.

Aceito em

$11 / 04 / 2021$. 


\section{INTRODUCTION}

Au début du nouveau millénaire, l'Assemblée Générale des Nations Unies a décidé de lancer une réflexion sur la question de la biodiversité au-delà de la juridiction nationale (BADJN) (TILLER et al., 2019). Après plus d'une dizaine d'années de discussions et négociations, l'Assemblée Générale a appuyé le lancement des négociations en vue d'un instrument contraignant pour la conservation de la BADJN, menacée de manière croissante par les activités humaines, I'une des expressions de l'Anthropocène (BIERMANN, 2014; VIOLA, BASSO, 2016).

La nature de res nullius des ressources biologiques en haute mer $^{1}$ a donné naissance à une obligation importante entre les États, celle de coopérer et de veiller sur les opérations des leurs ressortissants dans cette zone, de manière à garantir l'utilisation durable de la biodiversité et de prévenir les effets néfastes de son exploration et exploitation (OLIVEIRA, MALJEAN-DUBOIS, 2015; TANAKA, 2015). Ainsi, les négociations de New York sont la scène où on voit le développement de ces obligations.

Localisé en Atlantique Sud et possesseur d'une immense côte et d'une population dépendant de la santé des océans pour leur soutien, le Brésil a une grande responsabilité comme acteur dans ces négociations. Présentant une identité océanique qui est basée sur le fait que sa construction nationale est passée d'abord par la mer et par les relations et liens possibles par l'Atlantique (ABDENUR ; SOUZA NETO, 2014 ; MORAES, 2018 ; PINTO, 2019), ce pays peut beaucoup apporter dans les discussions et profiter également du processus pour définir un chemin du développement durable à l'échelle nationale.

Ainsi, cet article a comme objectif principal de dévoiler la position brésilienne dans les négociations pour la biodiversité marine des zones ne relevant pas de la juridiction nationale entre 2018 et 2019. Le choix de ces années a été fait pour mieux comprendre l'impact de la transition du gouvernement (de Michel Temer à Jair Bolsonaro) dans les négociations. La méthodologie adoptée est de nature essentiellement qualitative ; les données ayant été

\footnotetext{
${ }^{1}$ La BADJN peut aussi être nommée « biodiversité en haute mer » ou " biodiversité marine dans les zones ne relevant pas de la juridiction nationale ».
} 
collectées par l'étude des sources primaires et secondaires. Pour démontrer la position du pays, nous nous sommes appuyés sur les documents produits à l'occasion des négociations, principalement les IISD Reporting Services.

Après cette introduction, le texte est divisé entre quatre sections. La première aborde la question de la BADJN dans le droit international. La deuxième section présente les résultats des négociations BADJN, avec une attention particulière au positionnement brésilien. Nous discutons cette position dans la troisième section. La quatrième section contient les remarques finales.

\section{La BADJN dans le droit international}

Les océans ont été depuis des siècles au cœur des discussions entre les entités politiques, en raison de leur capacité de fonctionner comme un réseau au niveau global et de fournir des ressources importantes pour le maintien de la vie humaine. À titre d'information, le commerce mondial utilise les océans comme principale voie de transaction, environ $80 \%$ du total (UNCTAD, 2015). Les océans produisent environ 2,5 trillions de dollars par an, valeur qui dépend des conditions saines pour être atteint et conservé (HOEGH-GULDBERG, 2015). Bien sûr, les océans sont d'importantes sources de biens économiques, mais aussi essentiels pour la reproduction de la vie culturelle et sociale (KLEIN ; MACKENTHUN, 2012). Par conséquent, la valeur des océans ne peut pas être calculée simplement en prenant en compte leur valeur comme une marchandise ou un bien. Il faut inclure leur valeur inhérente et intrinsèque, ce qui est fait de manière variée entre les différents acteurs (BOWMAN ; DAVIES ; REDGWELL, 2010).

Outre la discussion philosophique, cette valeur (quoi qu'il en soit) ne peut être maintenue que par la conservation et l'utilisation durable des ressources marines, autrement dit le maintien de la "santé de la mer ", menacés par des activités humaines de plus en plus intensives (OECD, 2019 ; UNEP, 2019a). Les pressions d'origine anthropiques sont le résultat d'une « course aux océans » croissante (BARROS-PLATIAU, 2015) : les acteurs étatiques et nonétatiques perçoivent les ressources marines comme une nouvelle frontière de l'action humaine.

La biodiversité marine est l'une des ressources les plus fragiles rencontrées dans la mer, particulièrement au-delà des juridictions nationales, où les obligations des États pour sa 
conservation et utilisation durable sont beaucoup plus faibles que la biodiversité située sous les juridictions nationales (TANAKA, 2015). Ce décalage entre la protection internationale de ces ressources et leur utilisation croissante a emmené les acteurs internationaux à la création de nouveaux cadres de gouvernance pour la question. De cette façon, l'objectif de cette partie est de comprendre les règles qui définissent les enjeux de la biodiversité au-delà des juridictions nationales.

\section{La biodiversité en haute mer}

Pour comprendre la biodiversité au-delà des zones de juridiction nationale, il faut remarquer que la CNUDM (Convention des Nations Unies sur le Droit de la Mer) définit différentes zones maritimes au niveau global, pour lesquelles différents régimes juridiques s'appliquent. Ce n'est pas le but de cet article de faire une présentation complète des régimes applicables aux différentes zones dans la CNUDM, mais il faut les mentionner pour comprendre le statut de la biodiversité marine dans la « Constitution des Océans ».

Il est possible de distinguer les zones maritimes en deux groupes : celles sous la juridiction d'un État et celles qui ne le sont pas. Ricard (2017, p. 26-27) synthétise ces différences pour la première catégorie :

la CNUDM définit en premier lieu les eaux intérieures, adjacentes au territoire de l'État riverain qui y exerce sa souveraineté, puis les eaux territoriales. Au-delà, la Convention fait une distinction entre le régime du sol et du sous-sol, qui constituent le plateau continental, et celui de la colonne d'eau, la Zone Économique Exclusive (ZEE). Ces deux zones s'étendent jusqu'à la limite de deux cents milles nautiques. Le plateau continental étant considéré comme le " prolongement naturel " de son territoire terrestre, l'État y exerce des droits souverains exclusifs aux fins de l'exploration et de l'exploitation des ressources naturelles. Celui-ci peut, en outre, s'étendre au-delà de deux cents milles nautiques, jusqu'à trois cent cinquante milles des lignes de bases. Dans sa ZEE, l'État possède des droits souverains et exclusifs pour l'exploration, l'exploitation, la conservation et la gestion des ressources naturelles, biologiques et non biologiques. Dans ces deux espaces, l'État ne doit pas entraver de manière injustifiable les droits des autres États et est responsable de la protection de l'environnement. 
Image 1 - Délimitation des zones maritimes selon la CNUDM.

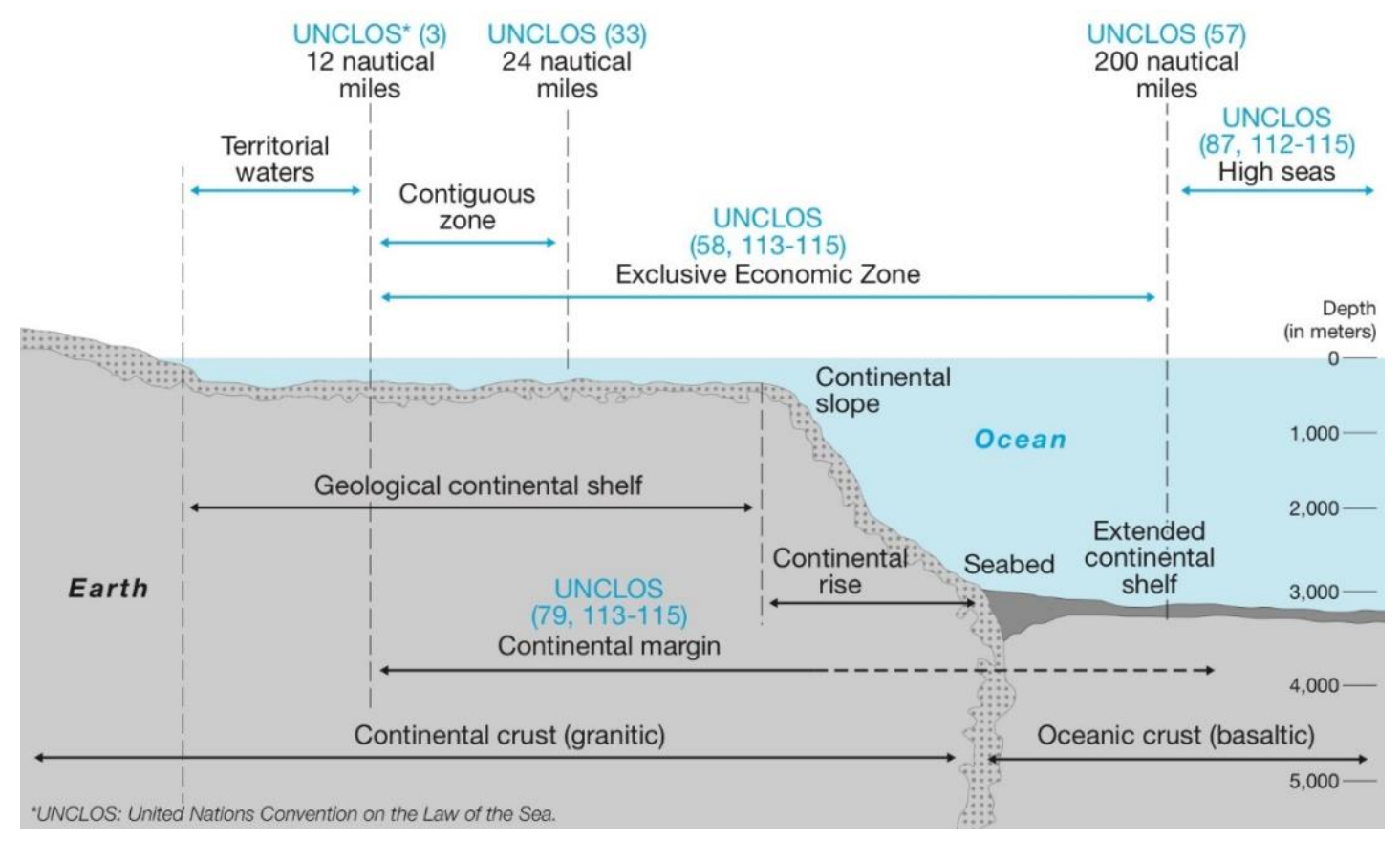

Fonte: Caron (2011).

Tanaka (2015) caractérise ces zones à partir de deux autres catégories, celles de la souveraineté territoriale et de la souveraineté spatiale. La souveraineté territoriale se caractérise par l'exclusivité de la juridiction de l'État, concernant toutes les eaux jusqu'à la ZEE (zone économique exclusive). La souveraineté spatiale comprend seulement les droits souverains de l'État, comprenant la ZEE et le plateau continental. Par contre, Moraes (2019) défend bien que cette distinction soit « juridiquement correcte... », elle est « ... politiquement illusoire, car elle exprime une différence que dans la pratique est minuscule $»^{2}(p .7$, traduit par l'auteur), car les pays souvent étendent la norme de la souveraineté (souveraineté territoriale) même aux espaces pour lesquels la CNUDM ne leur confère que des droits souverains.

Au-delà de ces aires, deux autres régimes s'étendent : la haute mer et la Zone. Le régime de la haute mer, défini dans la Partie VII de la CNUDM, comprend « toutes les parties de la mer qui ne sont comprises ni dans la zone économique exclusive, la mer territoriale ou les eaux intérieures d'un État, ni dans les eaux archipélagiques d'un État archipel », selon

\footnotetext{
${ }^{2}$ Original: « legally correct... politically misleading, as it exaggerates a difference that in practical terms is tiny ».
} 
l'article 86 (CNUDM, 1994, p. 38). Le régime de cette zone est fondé sur le principe de la liberté en haut mer ${ }^{3}$, où aucun État n'a le droit d'exercer sa souveraineté, mais où tous possèdent la liberté de mener différentes activités, mentionnées par la CNUDM à l'article 87. À son tour, la Zone est basée sur le principe du patrimoine commun de l'humanité $(\mathrm{PCH})^{4}$. L'Autorité Internationale des Fonds Marins (AIFM) est la responsable du contrôle et de l'organisation des activités conduites dans cette zone (RICARD, 2017). Selon Tanaka (2015), les limites de la Zone « consistent au maximum en 200 milles nautiques à partir de la ligne de base ou de la limite de la marge continentale lorsqu'elle s'étend au-delà de 200 milles nautiques $»^{5}$ (p. 178, traduit par l'auteur).

Finalement, il faut mentionner que les ressources visées par la CNUDM pour la Zone consistent en « toutes les ressources minérales solides, liquides ou gazeuses in situ qui, dans la Zone, se trouvent sur les fonds marins ou dans leur sous-sol, y compris les nodules polymétalliques ", selon l'article 133 (CNUDM, 1994, p. 51). On voit donc que la biodiversité ne fait pas partie des ressources de la Zone, à cette raison que le terme « biodiversité en haute mer » est beaucoup utilisé. Même qu'elle ne soit pas partie de la Zone dans les termes légaux, Il s'agit d'une zone riche en biodiversité, comme c'est le cas des sources hydrothermales (UNEP, 2019a).

Une critique faite à la Convention est que son approche zonale ne prend pas en compte I'unicité écosystémique des océans et de sa biodiversité. II est clair notamment que " les poissons " ne respectent pas les frontières politiques. Pour cette raison, l' « Accord aux fins de l'application des dispositions de la CNUDM relatives à la Conservation et à la Gestion des Stocks de Poissons dont les déplacements s'effectuent tant à l'intérieur qu'au-delà des EEZ

\footnotetext{
3 Bederman (2012) détaille l'évolution de cette idée depuis son émergence.

${ }^{4}$ Ce principe fait partie du corpus de l'CNUDM en régissant le régime de la Zone. D'après Tanaka (2015), le principe est un essai de donner le statut d'acteur international au sujet « humanité », donc porteur des valeurs et droits à être protégés, et de cette manière promouvoir l'intérêt commun de l'humanité, ce qui est au côté opposé des fondements des principes de souveraineté et des libertés en haute mer. Morin et Orsini (2014) pointent cinq caractéristiques de ce concept : d'abord, l'appropriation des espaces couverts par le principe est interdite ; il y a une obligation de coopérer pour l'utilisation durable des ressources couvertes; il faut avoir le partage équitable et juste des bénéfices de l'exploration des ressources PCH ; les espaces de PCH sont d'utilisation pacifique; et il faut compter sa conservation pour les générations futures.

${ }^{5}$ Original: « consist in at the maximum the 200 nautical miles from the baseline or the limit of the continental margin where it extends beyond 200 nautical miles $"$.
} 
(stocks chevauchants) et des stocks de poissons grands migrateurs " a été négocié et adopté par les États, puisqu'il existait cette difficulté de délimitation de droits de pêche par rapport aux zones et aux mouvements des stocks. La gestion de la pêche présente les règles pour la BADJN les plus développées, soit par hard law ou soft law ${ }^{6}$, accouplé avec l'obligation de coopérer pour que ces ressources ne s'épuisent pas (OLIVEIRA ; MALJEAN-DUBOIS, 2015), mais elle est aussi l'une des activités le plus souvent affectant la biodiversité marine (RICARD, 2017).

\section{Les négociations sur la BADJN}

Les zones situées au-delà des juridictions nationales représentent environ la moitié de la planète, mais leur gouvernance est marquée par une grande fragmentation, ce qui rend difficile faire face aux défis de la conservation et utilisation durable de ces ressources d'une manière cohérente et intégrée. Pour le cas de la BADJN, ce problème est beaucoup plus

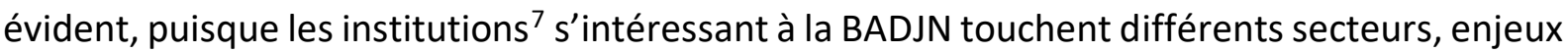
et ont même des localisations géographiques différentes (DRUEL, 2012). De plus, des questions relatives à la recherche scientifique et la bioprospection et d'autres activités liées à l'utilisation de ces ressources et aux impacts sur la conservation de la biodiversité en haute mer sont toujours marquées par un vide réglementaire (BECKMAN et al., 2018; UNEP, 2019a).

D’autre part, les activités humaines en haute mer se développent rapidement. La bioprospection, l'exploitation minière, la pêche, la recherche scientifique, le réseau mondial pour le transport par les voies maritimes ne reflètent qu'une partie d'une myriade d'activités qui, si non réglementées, peuvent entraîner des résultats catastrophiques sur le milieu marin et, par conséquent, menacer leur propre continuité. Même lorsque ces activités sont réglementées, leurs effets anthropiques persistent, avec la surpêche, la pollution provenant de différentes sources, l'acidification des océans ou encore le changement climatique comme

\footnotetext{
${ }^{6}$ C'est le cas du Code de Conduite pour une pêche responsable (FAO, 1995).

${ }^{7}$ Keohane (1988) caractérise les institutions comme « a general pattern or categorization of activity or to a particular human-constructed arrangement, formally or informally organized » (p. 383), alors que celui est une catégorie plus vaste et comprise mêmes les régimes.
} 
exemples (PERSHING, 2018 ; UNEP, 2019a).

Avec ces deux questions en tête, l'Assemblée Générale des Nations Unies (AGNU) a lancé en 2004 le «Groupe de travail spécial officieux à composition non limitée chargé d'étudier les questions relatives à la conservation et à l'exploitation durable de la biodiversité marine dans les zones situées au-delà de la juridiction nationale ", aussi référé comme " Groupe de travail BADJN ». Son mandat incluait les responsabilités :

a) de recenser les activités passées et présentes de l'Organisation des Nations Unies et des autres organisations internationales compétentes concernant la conservation et l'exploitation durable de la biodiversité marine dans les zones situées au-delà de la juridiction nationale; b) d'examiner les aspects scientifiques, techniques, économiques, juridiques, écologiques, socioéconomiques et autres de ces questions; c) d'identifier les principaux enjeux et les questions devant faire l'objet d'études plus poussées pour faciliter leur examen par les États ; d) d'indiquer, le cas échéant, les solutions et méthodes permettant de promouvoir la coopération et la coordination internationales pour la conservation et l'exploitation durable de la biodiversité marine dans les zones situées au-delà de la juridiction nationale (NATIONS..., 2004, p. 14).

Le groupe s'est réuni à plusieurs reprises de 2006 à 2015 et les positions ont évolué lentement jusqu'à $2011^{8}$, lorsque les États se sont mis d'accord sur le besoin de création d'un instrument international contraignant pour la BADJN, à condition que les contenus de cet instrument soient traités comme un «package ». Les avancées sur les questions des ressources marines génétiques (RMG), des aires marines protégées ( $A M P)$, des études d'impact environnementales (EIA) et du renforcement des capacités et transfert de technologies $(R C \& T T)^{9}$ doivent être acceptés par les États malgré certaines lacunes, en raison de la décision relativement favorable d'autre(s) question(s), pas nécessairement lié(es) (DRUEL et al., 2012). Cependant, c'est seulement dans la déclaration finale de la conférence Rio+20, en 2012, "L'avenir que nous voulons ", qu'il a été fixé la date finale pour décider de

\footnotetext{
${ }^{8}$ Le Groupe s'était réuni seulement trois fois entre 2006 et 2010, avec des désagréments qui ont marqués les discussions. Pour un rapport sur l'évolution de la substance de ces réunions, voir Druel et al. (2012).

${ }^{9}$ D'après le Groupe de Travail BADJN (NATIONS..., 2011) : " les ressources génétiques marines, y compris les questions liées au partage des retombées de l'exploitation de ces ressources, des mesures telles que les outils de gestion par zone, y compris des zones marines protégées, les évaluations d'impact sur l'environnement, le renforcement des capacités et le transfert de technologie marine » (p. 2).
} 
la création de cet instrument ${ }^{10}$, soit avant la fin de la $69^{\circ}$ session de l'AGNU, en 2015.

De cette façon, en 2015, le groupe de travail BADJN a finalement recommandé à I'AGNU de relancer les négociations pour un nouvel instrument international contraignant (NATIONS..., 2015a). À partir de la résolution 69/292 de l'AGNU, en 2015, s'est créé un Comité préparatoire qui a tenu quatre réunions, la dernière en juillet 2017 (NATIONS..., 2015b). Son résultat final a été l'élaboration d'un rapport qui souligne des possibilités pour le nouvel accord et contient des recommandations à l'AGNU (RICARD, 2017). En $2018^{11}$ les négociations ont commencé, après plus d'une dizaine d'années de discussions.

Ce régime pour la BADJN ne sera pas mis en œuvre lors de l'entrée en vigueur de cet instrument, après qu'il sera négocié, accepté, signé et ratifié. Bodansky (2010) explique que « dans la plupart des régimes environnementaux, le traité lui-même ne représente que la pointe de l'iceberg normatif " (p. 155, traduit par l'auteur), alors même que ce régime ${ }^{12}$ présente une existence limitée, puisque les règles générales et parfois spécifiques pour la BADJN sont dispersées parmi différents accords soit sous-régionaux, régionaux ou globaux, qui ne sont pas toujours coordonnées entre eux. De ce fait, ce nouveau traité promet justement d'être le nodule central permettant de regrouper tous ces efforts en direction d'une action globale plus précise et efficace.

\section{Le Brésil dans les négociations de la BADJN}

Les deux négociations de la « Conférence intergouvernementale chargée (CIG) pour l'élaboration d'un instrument international juridiquement contraignant (IIJC) se rapportant à

\footnotetext{
${ }^{10}$ Dans la Déclaration : " Nous appuyant sur ces travaux, nous nous engageons à nous attaquer d'urgence, avant la fin de la soixante-neuvième session de l'Assemblée générale, à la question de la conservation et de l'exploitation durable de la diversité biologique marine dans les zones qui ne relèvent pas des juridictions nationales, notamment en prenant une décision sur l'élaboration d'un instrument international dans le cadre de la Convention des Nations Unies sur le droit de la mer » (NATIONS..., 2012, p. 35)

11 Il faut noter que les délégués ont aussi réalisé une réunion d'organisation, au siège des Nations Unies à New York du 6 au 18 avril 2018.

$12 \mathrm{Il}$ y a la question si le régime pour le BADJN est déjà existant, alors un régime formel, ou un quasi-regime. Selon Carvalho (2012), ce dernier est marqué par une convergence minimale autour des exigences d'une politique internationale pour la BADJN et aussi un ensemble de principes qui est faible mais rapproche les arrangements internationaux d'un régime international, dans les mots de l'auteur : " an intermediate locus between the existence and nonexistence of a regime $»(p .145)$.
} 
la Convention des Nations Unies sur le droit de la mer et portant sur la conservation et I'utilisation durable de la biodiversité marine des zones ne relevant pas de la juridiction nationale » se déroulent à deux différents moments de la politique extérieure brésilienne. La première période s'est déroulée jusqu'en 2018, soit avant les résultats des élections générales ${ }^{13}$. Il faut souligner le fait que même avant de prendre ses fonctions, Président élu Bolsonaro a influencé les positions brésiliennes en politique extérieure. C'est le cas du rejet du Brésil du Pacte des migrations sûres, ordonnées et régulières (FELLET, 2019), à travers de ses déclarations de non-participation du traité dans son administration. La deuxième se déroule après les élections et la mise en place de la réforme ministérielle du gouvernement de Bolsonaro. Cela explique les différences de positions, voire les probables contradictions, en comparaison avec la première Conférence et les suivantes.

Dans cette partie, on s'appuiera sur les deux CIG pour I'IIJC, soit la CIG-1 de 4 à 17 septembre 2018, à New York; et la CIG-2 du 25 mars au 5 avril 2019, tenue également à New York.

La position brésilienne peut être comprise à l'aide de deux cadres généraux : I'un, ses positions individuelles, l'autre, ses positions comme partie d'un groupe. Concernant la première catégorie, il est clair que les discours gouvernementaux, les documents officiels et positionnements de la délégation brésilienne pendant la CIG, la reflètent bien. S'agissant de la deuxième catégorie, il est beaucoup plus difficile de comprendre en quoi les positionnements de ces groupes reflètent ou pas la politique extérieure brésilienne. Pour cette catégorie, on ne peut pas prendre en compte que les positionnements explicites du groupe pendant les négociations. On considère que le Brésil est toujours d'accord avec elles, sauf s'il les rejette expressément. Aussi, il y a la possibilité que les groupes que le Brésil participe aient des positions contraires voire contradictoires. En ce cas (un seul dans cette étude), on considère le groupe plus proche du Brésil comme partie de sa position.

Dans les négociations, on peut identifier deux groupes auxquels le Brésil participe et

\footnotetext{
13 En 2018, les brésiliens ont été appelés pour élire les chefs de l'exécutif des états fédéraux (27) et de la Fédération (le président, chef d’État et du Gouvernement), bien comme les députés fédéraux (512) et deux tiers des sénateurs (54 de 81).
} 
dont sa position peut être extraite : le Groupe des 77 et Chine ${ }^{14}$ (G77/Chine, pour la CIG-1 e CIG-2) et le "Like Minded Latin American Countries " ${ }^{15}$ (LMLAC, pour la CIG-2). Une des limites méthodologiques pour ce travail tient à la cartographie des alliances brésiliennes avec des groupes (soit avec acteurs publics ou privés), en tenant compte que nous n'avons pas eu l'accès aux négociations in loco, seulement à des transcriptions, des documents officiels et des discours. II reste à se demander si les groupes comme le BRICS ${ }^{16}$, IBAS $^{17}, \operatorname{CPLP}^{18}$ et d'autres sont mis en place pendant les négociations et, si c'est le cas, comment. En fin, il faut mentionner que les positions brésiliennes sont de caractères divers, puisque les négociations traitent des questions procédurales et substantives.

Les discussions dans les deux CIG ont été placées des groupes de travail, aux mêmes questions accordés en 2011 dans le "package ", soit : AMP, EIA, RC \& TT et RMG. On ajoute une autre catégorie, pour les questions générales, comme des déclarations spontanées.

La construction de la section 3.1.1 et 3.1.2 est basé sur les documents produits par I'IISD (IISD, 2018a; IISD, 2018b; IISD, 2019a; IISD, 2019b), qui ont couvert tous les aspects de la négociation, le Treaty Tracker de la High Seas Alliance (TREATY..., s.d.) et la base de donnés Papersmart des Nations Unies (NATIONS..., s.d.).

\section{CIG-1 : G77/Chine et le Brésil}

Pour la première question, les outils de gestion par zone (OGZ), incluant les aires marines protégées, le G77/Chine a mis en évidence la nécessité de reconnaître les OGZ déjà existants et a aussi appuyé une approche globale pour les OGZ, incluant des principes bien reconnus comme l'approche écosystémique ou la précaution. Le Groupe a aussi souligné le

\footnotetext{
${ }^{14}$ Le G77/Chine comme un groupe multi-divers et représentant de plus de 130 États fait face à un défi exceptionnel, celui de se positionner d'une manière stratégique pour que ses membres continuent à voir dans le groupe un porte-parole légitime. De cette façon, le groupe essaye d'être très actif dans les négociations, en appuyant une position qui garantisse les droits des États parties du groupe.

${ }^{15}$ Le groupe est formé par Argentine, Brésil, Colombie, Costa Rica, El Salvador, Équateur, Guatemala, Honduras, Mexique Panama, Paraguay, Pérou, République Dominicaine et Uruguay.

${ }^{16}$ Groupe formé par le Brésil, Russie, Inde, Chine et Afrique du Sud.

${ }^{17}$ L'abréviation pour le Forum de Dialogue Inde, Brésil et Afrique du Sud.

${ }^{18}$ À la Déclaration de Brasília, dans la IX Conférence des Chefs d'État et de Gouvernement de la Communauté des pays de langue portugaise, les pays ont soutenu leur compromis avec les négociations de l'IIJC (MINISTÉRIO..., 2016).
} 
besoin de contrôle de la mise en œuvre de ces outils, un travail qui serait effectué par un organe scientifique/technique, la Conférence des Parties (CdP) s'occupant du suivi.

Le Brésil a été bien actif dans ces discussions ${ }^{19}$. Dans le même sens que le G77/Chine, le Brésil a marqué sa préférence pour une approche globale, mais sans préjudice vis-à-vis des institutions existantes; l'inclusion d'un comité scientifique/technique, soutenu par des experts indépendants, pour les OGZ; en ajoutant que les décisions doivent être prises par une $\mathrm{CdP}$, la mise en œuvre étant sous la responsabilité de l'État du pavillon. Le Brésil a soutenu quelques positions des autres pays relatives aux propositions pour les OGZ, soit : que ces propositions peuvent venir des Parties à la future Convention et de différents organes (comme le comité scientifique), que les organismes déjà existants et les États puissent le faire individuellement ou conjointement, en n'oubliant pas les États côtiers dans ce processus de proposition.

Le G77/Chine avait exprimé que le groupe voulait examiner une liste provisoire des activités nécessitant des EIE. Après cette position, le Brésil a montré une position contraire : il s'opposait à l'inclusion d'une liste. Cela montre que faire partie d'un groupe ne reflète pas l'alignement automatique et que le pays peut s'exprimer d'autre façon à la base de sa souveraineté.

Comme membre du Système du Traité sur l'Antarctique, le Brésil a rappelé le rôle que son Protocole relatif à la protection de l'environnement pourrait jouer comme source d'inspiration, ainsi que la CNUDM pose des directives pour le suivi et l'EIE à ses articles 204 à 206.

Le G77/Chine est beaucoup intervenu sur les EIE. II a préconisé la transparence, la participation, l'utilisation des meilleures informations scientifiques disponibles, en tenant compte les connaissances traditionnelles, et s'est prononcé pour un organe qui autorise les activités et soit en charge du suivi et de l'examen. S'agissant du contenu, ce groupe a aussi proposé un résumé et une description non technique, appelé RIMA au Brésil (Relatório de

\footnotetext{
${ }^{19}$ Le Brésil, par exemple, a proposé la création d'un sanctuaire pour les baleines au Atlantique Sud en 2018 à travers de la Commission Baleinière Internationale, mais la proposition n'a pas été accepté (MATSUURA ; BAIMA, 2018).
} 
Impacto Ambiental, ou Rapport d'impact environnemental, en traduction libre), parmi d'autres questions, incluant les effets cumulatifs et les impacts socio-économiques. La définition proposée pour l'EIE inclut la prise en compte des impacts socio-économiques, culturels et sur la santé humaine. Les principes défendus pour les EIE reflètent les positions précédentes du groupe, comme les principes du pollueur-payeur, l'approche écosystémique, l'approche scientifique et l'approche de précaution, entre autres. Enfin, le groupe défend que les EIE ne soient pas nécessaires s'il existe déjà des obligations pour l'activité couverte.

Reflétant un enjeu très important pour les pays du G77/Chine et pour le Brésil, le renforcement des capacités et le transfert de technologies sont une manière de combler l'écart existant entre les pays détenteurs de technologies pour l'exploration de la haute mer et les autres ${ }^{20}$. Le Brésil a défendu la référence aux équipements marins et au partage des infrastructures. Il s'est aussi inspiré de son expérience de l'organisation de la conférence de Rio+20 et a suggéré de développer une plate-forme virtuelle pour que les États puissent y préciser leurs priorités et besoins et, ainsi, les évaluer ${ }^{21}$.

Le G77/Chine a défendu le cas spécial des pays en développement de différentes catégories, et le besoin du renforcement de la recherche scientifique marine et des capacités technologiques, d'infrastructure et d'équipement, et a mis en évidence la possibilité de duplication des efforts, comme lesquelles provenus de l'Autorité Internationale de Fonds Marins (AIFM). De cette façon, le groupe a appuyé un mécanisme possible d'accès à l'information et de diffusion, en ajoutant la possibilité de créer un référentiel central pour les données de base et pour les demandes de RC \& TT, au cas par cas. N'oubliant pas le financement, le groupe a aussi rappelé sa nécessité et a prôné la création d'un fonds d'affectation spéciale pour le partage des avantages.

La dernière question, celle de ressources marines génétiques, est celle qui montre le plus les divisions entre les pays du Nord et du Sud, où les pays les plus avancés

\footnotetext{
20 Juniper (2013) explique qu'il est nécessaire des infrastructures pour la bioprospection en haute mer, et l'action dans cette zone en général, principalement des bateaux de 60 mètres en environ, ceux qui sont concentrés aux pays teneurs des technologies plus avancés, soit les trois premier la Russie, I'Union Européenne et les États-Unis. ${ }^{21}$ Les « Dialogues pour le Développement Durable » ont été une initiative brésilienne pour la Conférence, où des représentants de la société ont pu faire des recommandations directes pour la Conférence à travers la plateforme virtuelle créée spécialement pour la Rio +20 (BRASIL, 2012).
} 
technologiquement s'appuient sur le besoin de voir les RMG comme partie de l'idée de la liberté en haute $\mathrm{mer}^{22}$, tandis que les pays du Sud, représentés principalement pour le G77/Chine, sont favorables à les qualifier de patrimoine commun de l'humanité (PCH). Le Brésil se positionne en faveur de ce dernier principe, considérant que tous les États devraient avoir un accès équitable et juste aux RMG, en tant que $\mathrm{PCH}$. Cette position est soulignée dans le rapport de l'UNEP (2019b, p. 126, traduit par l'auteur) :

Les pays à faible revenu dont l'économie repose sur l'agriculture ne sont généralement pas à l'origine de la consommation accrue de ressources naturelles qui entraîne des effets négatifs sur l'environnement et la santé humaine dans le monde entier. Le milliard d'individus les plus riches représentent $72 \%$ de la consommation des ressources mondiales, tandis que le 1,2 milliard de personnes les plus pauvres n'en consomment que $1 \% .^{23}$.

C'est une position qui montre les écarts entre les pays sur l'utilisation de ces ressources, où le $\mathrm{PCH}$, une fois défini par un accord international, garantissait l'accès équitable et juste à la BADJN.

Le pays a aussi défendu l'inclusion des dérivés des RMG, déjà que ces considérations sont incluses au Protocole de Nagoya et qu'il n'existe pas donc aucune raison pour qu'il ne le soit pas à l'IIJC ; la consultation dans le cas de chevauchement de RMG ; la réglementation de l'accès aux RMG, inclus la divulgation de l'origine et du but (ce que le G77/Chine défend aussi). Une proposition brésilienne intéressante a aussi été mise en place, c'est que les obligations de partages des avantages (selon lesquelles tous les pays seraient inclus, principalement les pays en développement et les moins avancés) soient réalisées à partir d'un fonds mondial composé du $1 \%$ du revenu net du résultat de la commercialisation des RMG à mettre en place.

II faut aussi mentionner l'évolution de la position traditionnelle du G77/Chine sur le PCH par rapport aux RMG pendant les négociations. Le groupe a proposé que l'exploitation des RMG devrait être régie par le $\mathrm{PCH}$, tandis que l'accès à celles-ci serait régi par le principe

\footnotetext{
${ }^{22}$ Le cas exceptionnel de l'Union Européenne, elle s'est située au milieu, puisqu'elle ne reconnaît pas que les RMG sont patrimoine commun de l'humanité, mais n'agrée pas à une liberté sans frontières et préfère une approche basée sur des principes d'équité (DRUEL et al., 2012).

${ }^{23}$ Original: " low-income countries with agriculturally based economies are not typically driving the increased consumption of natural resources that is leading to negative environmental and human health impacts around the world. The billion richest individuals account for 72 per cent of the consumption of global resources, while the poorest 1.2 billion consume only 1 per cent $»$.
} 
de liberté de la haute mer, selon une approche hybride qui pourrait réduire les divergences entre pays et faire avancer les négociations du traité. Mais cet accès serait réglementé, en tenant compte des RC \& TT. Le groupe a aussi suggéré la création d'un organisme mondial de normalisation pour les RMG, un régime d'accès et partage des avantages (monétaires et non monétaires) basé sur l'AIFM, le Protocole de Nagoya et le Traité international sur les ressources phytogénétiques.

Au-delà des questions des RMG in vitro et in situ, le groupe recommande l'inclusion des RMG in silico ${ }^{24}$. La question de la pêche est aussi mise sur la table ; le G77/Chine voudrait que le traité différencie les poissons comme produit de base et comme matériau génétique. C'est un des indices montrant qu'il n'y a pas la volonté que ce nouveau IIJC change les régimes de la pêche.

\section{La CIG-2 : le Brésil entre le G77/Chine et le LMLAC}

La Conférence a vu l'émergence d'un nouveau groupe pour l'Amérique Latine, sur lequel le Brésil s'est be aucoup appuyé pour ses positionnements. Si dans le premier CIG nous avons compté plus que quinze positionnements brésiliens dans les sources utilisés, dans le CIG-2 nous n'en comptons qu'une, dans laquelle le Brésil parle pour le LMLAC - pas pour luimême, comme dans la CIG-2. Par conséquent, nous n'avons pas une position uniquement brésilienne pour le CIG-2, mais seulement une position du Brésil comme partie de groupes.

La différence dans cette négociation c'est que la présidente de la Conférence, Rena Lee, a partagé un document appelé « Document établi par la Présidente pour faciliter les négociations ", qui contenait les options pour l'IIJC (NATIONS..., 2018). Le document est le reflet de la difficulté de la question négociée, puisqu'il contient presque toutes les options apportées par les États. D'un côté, le document a aidé aux discussions, car les délégations ont eu un point en commun pour en discuter. D'un autre côté, cela explique que les discussions aient été centrées sur les options du document, sans beaucoup innover. Les discussions ont

\footnotetext{
${ }^{24}$ Voir Gallezot (2002) pour la question du « in silico » dans la recherche des ressources génétiques.
} 
suivi le même format que lors de la CIG-1, avec des groupes de travail sur chaque thème du « package ».

Sur toutes les questions, les discussions ont été approfondies en relation avec la CIG1, grâce au document de la présidente. Les aires marines protégées ont aussi bénéficié de cet approfondissement.

Malgré la composition différente du G77/Chine et du LMLAC, il n'est pas atypique de voir les deux groupes défendre les mêmes positions sur les sujets de la négociation. Par exemple, sur la question des OGZ, les deux groupes ont appuyé une liste non exhaustive pour I'identification des aires, incluant les AMP, de façon à ce que le possible organe de décision de I'IIJC accepte ou non l'aire proposée. De plus, le LMLAC a préféré que la prise de décision se fasse par consensus.

Le G77/Chine a indiqué la limitation de prendre les OGZ seulement par les AMP, donc il faut avoir une cohérence entre les différents types d'OGZ. À son tour, le LMLAC a préféré un cadre mondial pour les OGZ qui complète les mesures des institutions régionales et sectorielles préexistantes. Il a aussi noté la nécessité de consultations entre les États, principalement entre États adjacents, et le besoin de révision constante et de surveillance par un comité de surveillance et compliance.

Sur les EIE, il y a eu aussi des positionnements contraires. Tandis que le G77/Chine a préféré reporter les discussions, le LMLAC a préféré préciser les pas nécessaires pour les EIE et a présenté des suggestions, basées sur les principes de responsabilité, transparence et d'autres, bien comme l'envoi des rapports de surveillance et révision à l'organe scientifique/technique. Sur la question de l'évaluation stratégique environnementale, le G77/Chine a préféré reporter et le LMLAC a souhaité d'en clarifier la portée. Mais il y a eu aussi des positionnements similaires, comme sur le besoin de détailler le contenu des EIE.

Deux dernières questions sont posées pour les EIE : les activités nécessitant une EIE et la relation de l'EIE avec d'autres instruments. Sur la première, le LMLAC a appuyé une liste ouverte des activités en annexe, la nécessité de tenir compte des impacts cumulatifs et de faire un EIE et d'évaluer les effets lorsque l'État considère que l'activité peut causer une pollution substantielle. Sur la deuxième question, le G77/Chine a dit que le nouvel instrument 
doit respecter et appuyer les instruments, tandis que le LMLAC a suggéré que l'IIJC centralise et qu'il pose les standards minimums, d'une manière que ces instruments soient en accord avec ce nouvel instrument.

La RC \& TT a été le sujet qui a donné lieu au plus de positions en commun entre les deux groupes par rapport aux types et modalités, au centre d'échange et au financement. Sur les types, les deux groupes ont défendu l'inclusion d'une liste des activités de RC \& TT. Le LMLAC a appuyé sa révision, évaluation et réglage périodique. Pour les modalités, les deux préfèrent les esquisser, le G77/Chine optant pour un langage fort en relation au transfert de technologies; le LMLAC optant pour un mécanisme au cas par cas, sans qu'il ne soit pas nécessaire son évaluation par un organe, et juste, sans que les droits de propriété intellectuelle soient un obstacle à sa réalisation. En relation au centre d'échanges, ils ont souligné ses fonctions. Pour le financement, les deux groupes sont d'accord sur le fait qu'il doit être volontaire et contraignant. Le G77/Chine a recommandé une liaison entre le centre et les banques de gènes. Le G77/Chine s'est encore positionné par rapport à deux autres questions : la surveillance et révision, dont il prône l'inclusion, et les catégories spéciales d'États, en soulignant le besoin de reconnaître que les Petits États Insulaires en Développement (PEID) sont confrontés à des circonstances particulières.

Les RMG sont toujours une question très délicate, mais c'est aussi à ce sujet que les États essaient de montrer leur vision pour combler les inégalités découlant de l'exploration de ces ressources. On peut évaluer les positions à partir de ces thèmes : l'accès, les avantages à partager, les bénéficiaires, les modalités de partage, les droits de propriété intellectuelle (DPI), la portée et la surveillance.

Par rapport à la question de l'accès, le LMLAC a soutenu que le traité devrait couvrir toutes les activités liées aux RMG, en soulignant le besoin d'éclaircir les conditions de sa promotion, en particulier quand il y a un changement de décision sur son utilisation. Sur la question des avantages à partager, le LMLAC a défendu le partage des avantages monétaires et non monétaires, incluant une liste ouverte, et a considéré que l'option de partage volontaire ne serait pas possible, trouvant ici une position en commune avec le G77/Chine. Ceux qui devraient recevoir les partages des avantages ne sont que les États, principalement 
les pays en développement, d'après le LMLAC. Le G77/Chine a mis en évidence, par contre, que le partage par soi n'est pas suffisant, et a aussi prôné que la question des DPI soit incluse dans cette section. Sur la portée, le G77/Chine a préféré que les activités de RMG respectent les droits de l'État côtier sur le territoire duquel elles seraient trouvées. L'autre position en commun entre les deux groupes a été sur la question de surveillance, dont ils ont appuyé son inclusion.

\section{Discussion}

Deux considérations sont saillantes : la nature fragmentée de la gouvernance globale pour la BADJN et une approche non-stratégique de la diplomatie brésilienne pour la BADJN et aussi pour les négociations.

Biermann et al. (2009) définissent la fragmentation en trois catégories (fragmentation synergique, coopérative et conflictuelle) basées sur trois variables: l'intégration institutionnelle, les conflits des normes et les types de réseaux d'acteurs. Pour qu'une fragmentation soit synergique, elle doit présenter une intégration forte avec un nodule central dans une institution qui rassemble les autres; les normes centrales doivent aussi être bien intégrées, sans conflits prédominants entre elles ; et il doit y avoir un support des acteurs aux institutions. Pour la fragmentation coopérative, on a différentes institutions pour la prise des décisions qui ne sont pas forcément intégrées, dont les relations entre les règles sont ambiguës et les institutions centrales ne rassemblent pas tous les pays importants pour la question. Finalement, la fragmentation conflictuelle présente le niveau le plus haut de fragmentation, marqué par l'absence d'institutions intégrées pour la prise des décisions voire de chevauchements où les normes sont clairement en conflit entre elles, faisant partie de différents régimes et, par rapport aux acteurs, leurs affiliations sont différentes et peuvent faire avancer ces conflits de normes.

L'existence d'une architecture de gouvernance fragmentée n'est pas synonyme des résultats sous-optimaux. Comme expliqué par Anselmi (2018), cette fragmentation peut même avoir un impact positif, par exemple, en permettant la conclusion des accords 
internationaux par petits groupes d'États (une caractéristique importante pour la gouvernance de la BADJN si on pense aux régions partagées, comme la Méditerranée ou I'Antarctique) et renforçant la participation et l'influence des acteurs non-gouvernementaux.

$\mathrm{Au}$ vu de nos recherches, la gouvernance de la BADJN relève de la fragmentation conflictuelle. La CNUDM est la constitution des océans, en apportant les règles centrales, pourtant il existe une myriade d'institutions et régimes sectoriels et régionaux, qui parfois ont même pour rôle d'affaiblir la CNUDM, comme c'est le cas de l'Accord relatif à l'application de la Partie XI de la CNUDM (OLIVEIRA ; MALJEAN-DUBOIS, 2015). Donc, le nouvel IIJC est au milieu de cet enjeu : soit il va s'ajouter à la fragmentation de cette gouvernance, soit il va s'adresser à l'intégration, en diminuant les effets pervers de la fragmentation.

Le Brésil, comme acteur dans cette gouvernance, a une responsabilité basée sur le droit international de coopérer, comme tous les autres États. Au-delà de cette raison générale, il faut réfléchir la place du Brésil dans le monde.

Pour les océans, il est clair que le Brésil se présente comme l'Amazonie Bleue, le vocabulaire utilisé pour définir sa ZEE. Dans sa vision, cette espace est aussi riche que l'Amazonie verte. Barros et al. (2015, p. 205) expliquent que

\begin{abstract}
certains y voient une stratégie d'intégration avec l'Amazonie verte absolument nécessaire pour le contrôle et la gestion de la région par rapport au crime organisé transnational, notamment au trafic de drogue et d'êtres humains. D'autres l'appréhendent dans le cadre d'une stratégie beaucoup plus large et plus ambitieuse. En assurant sa souveraineté sur ses ressources comme la coopération avec les voisins atlantiques, le Brésil entend jouer un rôle international plus important au XXIe siècle. Reste une minorité optimiste de ceux qui espèrent que le concept d'Amazonie bleue servira à renforcer la protection environnementale.
\end{abstract}

Le pays présent donc une approche centrée sur sa juridiction nationale par rapport aux ressources marines ${ }^{25}$, selon laquelle la Marine est le nodule central pour la gouvernance des océans au Brésil, par conséquent avec une approche militaire, au but de protéger des intérêts et ressources brésiliens, soit garantir sa souveraineté (SILVA, 2017). Selon Moraes (2019), les espaces marins ont été pris comme une possibilité de prolongation de la souveraineté, et cette

\footnotetext{
25 Il faut mentionner que le pays mène deux autres projets pour les ZADJN : le PROAREA, pour mener les activités minières à la Zone, et le PROANTAR, pour les activités de l'Antarctique (SECRETARIA..., s.d.).
} 
approche est bien celle utilisée par le Brésil. II n'existe donc pas de position brésilienne sur la biodiversité au-delà de la juridiction nationale aussi forte que pour les ressources sur sa juridiction. Autrement dit, l'attention du pays ne pèse pas encore sur les ZADJN.

Pour trouver les sources des positions brésiliennes apportées aux deux CIG, on se réfère à Rodrigo de Moraes qui souligne les incitations à se positionner comme tel :

(...) le Brésil est fortement incité à mettre l'accent sur la redistribution des ressources et de la technologie. Comme les institutions brésiliennes ont une capacité limitée de recherche et d'exploitation des ressources dans des zones ne relevant pas de la juridiction nationale, un système de redistribution augmenterait la probabilité de partenariats internationaux et de transfert de technologie. Toutefois, ce n'est pas ce que la plupart des pays développés proposent, car ils préfèrent un régime de type " liberté des mers ». Cela permettrait à quelques institutions de ces pays d'effectuer des recherches dans des zones situées au-delà de la juridiction nationale, qui représentent $64 \%$ de la surface des océans et $95 \%$ de leur volume. En outre, le Brésil étant l'un des rares pays mégadivers, l'accent mis sur la protection de l'environnement peut être utilisé comme un avantage comparatif, compensant partiellement les faiblesses dans d'autres domaines ${ }^{26}$ (QUEIROZ, 2019a, online, traduit par l'auteur).

On voit que ces considérations reflètent principalement les positionnements du Brésil lors de la CIG-1, mais aussi dans groupes dont il fait partie, même si les groupes ne reflètent pas seulement des idées brésiliennes. La préoccupation principale est donc de ne pas être laissé à la traîne, en garantissant un partage égal de ces ressources et de leur utilisation, en tant que patrimoine commun de l'humanité. Ainsi, on explique sa préférence envers une approche globale de la BADJN, ce qui garantirait de rassembler toutes les activités liées à la question dans un forum auquel le pays aurait accès et disposerait d'une voix.

En revanche, il y a une considération relative à l'absence de positionnement individuel de la délégation brésilienne à la CIG-2. Le Brésil a fait le choix de s'exprimer plutôt à travers des groupes, pour des raisons encore obscures. Par contre, on peut imaginer que cette décision trouve son origine dans le changement radical promis par la nouvelle administration

\footnotetext{
${ }^{26}$ Original: «...Brazil has strong incentives to emphasize redistribution of resources and technology. As institutions in Brazil have limited capacity to do research and exploit resources in areas beyond national jurisdiction, a redistributive system would increase the likelihood of international partnerships and transfer of technology. This is not what most developed countries are proposing, however, as they prefer a 'freedom of the seas' type of regime. This would allow a few institutions in these countries to do research in areas beyond national jurisdiction, which comprise $64 \%$ of the surface of the oceans and $95 \%$ of its volume. Furthermore, as Brazil is one of the few megadiverse countries, a focus on environmental protection can be used as a comparative advantage, partially compensating for weaknesses in other areas ».
} 
en matière de politique extérieure, avec une moindre place pour les questions environnementales ${ }^{27}$.

Le résultat final de I'IIJC ne peut pas être envisagé maintenant, alors que les négociations sont encore en cours - la dernière négociation a été reportée en raison de la pandémie du Covid-19. A l'image de l'effet papillon, où de petits changements dans les conditions initiales peuvent produire de grandes conséquences dans des systèmes complexes (HO, 2017), le futur est incertain. Par contre, reconnaître cette limitation n'est pas être soumis aux flux. Il faut se préparer pour les chemins qui pourraient être ouverts, à partir de la construction d'une position stratégique, c'est-à-dire, comme on a proposé dans une autre étude, construire une diplomatie stratégique (QUEIROZ, 2019b).

\section{Remarques finales}

Le nouveau tournant de la politique extérieure brésilienne promis par le président Bolsonaro offre des perspectives de changements profonds pour la tradition diplomatique brésilienne. Deux phénomènes sont saillants: une position discursive contre l'« ordre globaliste " et un alignement presque automatique sur les États-Unis. Comme I'ont bien remarqué Basso et Viola (2018), la première position se traduit pour être contre ce qu'on appelle de "gouvernance globale », pour l'environnement elle est essentielle, d'après que les problèmes environnementaux ne sont plus partis d'un territoire précis, mais partagé dans un monde complexe et interdépendant, où les solutions doivent partir des actions concertées. Pour la BADJN, cette nécessité est beaucoup plus claire, puisque comme on l'a déjà souligné elle n'est placée sous aucune juridiction nationale, les États ayant l'obligation de coopérer pour sa conservation.

S'agissant de l'alignement, le Brésil risque de construire sa politique extérieure sur la BADJN non par rapport à ses besoins de développement durable, mais aux intérêts d'autres acteurs selon une nécessité de court-terme d'alignement à cause des intérêts de

\footnotetext{
27 II faut mentionner que le projet pour les ministères du président Bolsonaro ne comptait pas avec un Ministère de l'Environnement, mais après des pressions, il a décidé de le créer, bien que son rôle se voit limité, par rapport au renforcé MAPA (Ministère d'Agriculture, Élevage et Approvisionnement) (BRAGANÇA, 2018).
} 
" désidéologisation » - qu'au contraire de " désidéologiser », remplace une idéologie par une autre plus digestible aux acteurs gouvernementaux. II ne faut donc pas déconsidérer les relations du Brésil avec d'autres acteurs plus proches de sa réalité, ses besoins et aussi ses intérêts, d'une manière que c'est plus facile de travailler avec ces groupes d'États pour avancer ses propositions, mais il doit garder son autonomie et son indépendance exprimées par la construction d'une diplomatie stratégique.

Cette cartographie de la position brésilienne n'est qu'un travail préliminaire nécessaire pour la construction d'une position qui tienne compte des besoins internes et externes du pays. Comme Alons (2007), Milner (1997) et Putnam (1988) le remarquent, l'aspect domestique doit être pris en compte pour l'analyse intégrale des relations internationales. On en conclut qu'il faut continuer dans la direction de cet article, en ajoutant les dynamiques internes brésiliennes et les relations du Brésil avec l'extérieur (et vice-versa), ainsi que les impacts sur la construction de sa politique extérieure concernant la BADJN, en considérant le besoin d'une approche stratégique.

\section{RÉFÉRENCES}

ABDENUR, Adriana Erthal; SOUZA NETO, Danilo Marcondes de. O Brasil e a cooperação em defesa: a construção de uma identidade regional no Atlântico Sul. Revista Brasileira de Política Internacional, v. 57, n. 1, 2014.

ALONS, Gerry. Predicting a State's Foreign Policy: State Preferences between Domestic and International Constraints. Foreign Policy Analysis, n. 3, p. 211-232, 2007.

ANSELMI, Marcela Braga. Conflictive climate governance architecture: an analysis of the climate negotiations under the international civil aviation organization (ICAO). 133p.

Dissertação (Mestrado). Instituto de Relações Internacionais, Universidade de Brasília, 2018.

BARROS-PLATIAU, Ana Flávia et al. Correndo para o mar no antropoceno: a complexidade da governança dos oceanos e a estratégia brasileira de gestão dos recursos marinhos. Revista de Direito Internacional, v. 12, n. 1, p. 149-168, 2015.

BARROS, Jorge Gomes do Cravo et al. « Amazonie bleue » et projection brésilienne sur l'avenir. Outre-Terre, n. 1, p. 204-212, 2015. 
BASSO, Larissa; VIOLA, Eduardo. A poor future for Brazil in Global Environmental Governance?, by Larissa Basso \& Eduardo Viola. Mundorama - Revista de Divulgação Científica em Relações Internacionais. Disponível em:

<https://www.mundorama.net/?p=24997>. Acessado em 10 de setembro de 2020.

BEDERMAN, David J. The sea. In: FASSBENDER, Bardo et al. (orgs.). The Oxford handbook of the history of international law. Oxford University Press, 2012.

BECKMAN, Robert C.; MCCREATH, Millicent; ROACH, Ashley; SUN, Zhen. High Seas Governance: Gaps and Challenges. Brill/Nijhoff, 2018.

BIERMANN, Frank. The Anthropocene: a governance perspective. The Anthropocene Review, v. 1, n. 1, 2014, pp. 57-61.

BIERMANN, Frank et al. The fragmentation of global governance architectures: A framework for analysis. Global Environmental Politics, v. 9, n. 4, p. 14-40, 2009.

BODANSKY, Daniel. The art and craft of international environmental law. Harvard University Press, 2010.

BOWMAN, Michael; DAVIES, Peter; REDGWELL, Catherine. Lyster's international wildlife law. Cambridge University Press, 2010.

BRAGANÇA, Daniele. Bolsonaro confirma promessa: Ministério do Meio Ambiente deixará de existir. O Eco, 2018. Disponível em: <https://www.oeco.org.br/noticias/bolsonaroconfirma-promessa-ministerio-do-meio-ambiente-deixara-de-existir/>. Acessado em $10 \mathrm{de}$ setembro de 2020.

BRASIL. Relatório Rio+20, o Modelo Brasileiro: Relatório de Sustentabilidade da Organização da Conferência das Nações Unidas sobre Desenvolvimento Sustentável. Brasília: Fundação Alexandre de Gusmão, 2012. Disponível em:

$<$ http://www.rio20.gov.br/documentos/relatorio-rio-20/1.-relatorio-rio-

20/at_download/relatorio_rio20.pdff>. Acessado em 10 de setembro de 2020.

CARON, D. Negotiating our future with the oceans. A planet for life, p. 25-34, 2011.

CNUDM. Convention des Nations Unies sur le droit de la mer. 1994. Disponível em: $<$ https://www.un.org/depts/los/convention agreements/texts/unclos/unclos f.pdf>. Acessado em 10 de setembro de 2020.

CARVALHO, Fernanda Viana de. The Brazilian position on forests and climate change from 1997 to 2012: from veto to proposition. Revista Brasileira de Política Internacional, v. 55, n. SPE, p. 144-169, 2012. 
DRUEL, Elisabeth; ROCHETTE, Julien; BILLÉ, Raphaël; CHIAROLLA, Claudio. A long and winding road: International discussions on the governance of marine biodiversity in areas beyond national jurisdiction. Studies N07/13, Paris : IDDRI, 2012.

FAO (Food and Agriculture Organization). Code de conduite pour une pêche responsable. Rome: FAO, 1995. Disponível em: <http://www.fao.org/3/a-v9878f.pdf>. Acessado em 10 de setembro de 2020.

FELLET, João. Em comunicado a diplomatas, governo Bolsonaro confirma saída de pacto de migração da ONU. BBC, 2019. Disponível em: <https://www.bbc.com/portuguese/brasil46802258>. Acessado em 10 de setembro de 2020.

GALLEZOT, Gabriel. La recherche in silico. In : CHARTRON, G. (org.). Les chercheurs et la documentation électronique : nouveaux services, nouveaux usages. Edition du cercle de la Librairie, Coll. Bibliothèque, juillet 2002. Disponível em:

$<$ https://archivesic.ccsd.cnrs.fr/sic_00177318v3/document>. Acessado em 10 de setembro de 2020.

HIGH Seas Alliance. Treaty Tracking. [s.I.], [s.d.]. Disponível em:

$<$ http://highseasalliance.org/treatytracker/>. Acessado em 10 de setembro de 2020.

HO, Peter. The Challenges Of Governance In A Complex World. World Scientific, 2017.

HOEGH-GULDBERG, Ove. Reviving the ocean economy: the case for action-2015. WWF International, Gland, Switzerland, 2015.

IISD (International Institute for Sustainable Development). Bulletin des Négociations de la Terre. IISD Reporting Services, vol. 25, n. 169, 2018a. Disponível em:

<http://enb.iisd.org/oceans/bbni/igc1/>. Acessado em 10 de setembro de 2020.

Bulletin des Négociations de la Terre. IISD Reporting Services, vol. 25, n. 179, 2018b. Disponível em: <http://enb.iisd.org/oceans/bbnj/igc1/>. Acessado em 10 de setembro de 2020.

Earth Negotiations Bulletin. IISD Reporting Services, vol. 25, n. 186a, 2019.

Disponível em: <http://enb.iisd.org/oceans/bbnj/igc2/>. Acessado em 10 de setembro de 2020.

Earth Negotiations Bulletin. IISD Reporting Services, vol. 25, n. 195b, 2019.

Disponível em: <http://enb.iisd.org/oceans/bbnj/igc2/>. Acessado em 10 de setembro de 2020.

JUNIPER, S. Kim. MGR in ABNJ: Clarifying terminology and constraining expectations. UN, 
Division for Oceans Affairs and the Law of the Sea, 2013. Disponivel em:

<http://www.un.org/Depts/los/biodiversityworkinggroup/workshop1_juniper.pdf Acessado em 10 de setembro de 2020.

KEOHANE, Robert O. International institutions: Two approaches. International studies quarterly, v. 32, n. 4, p. 379-396, 1988.

KLEIN, Bernhard; MACKENTHUN, Gesa. Sea changes: historicizing the ocean. Routledge, 2012.

KRASNER, Stephen. Structural causes and regime consequences: regimes as intervening variables. International Organization, v. 36, n. 2, 1982, pp. 185-205.

MATSUURA, Sérgio; BAIMA, Cesar. Proposta de criação do Santuário de Baleias do Atlântico Sul é rejeitada. Jornal O Globo, 2018. Disponível em:

<https://oglobo.globo.com/sociedade/ciencia/meio-ambiente/proposta-de-criacao-dosantuario-de-baleias-do-atlantico-sul-rejeitada-23058442>. Acessado em 10 de setembro de 2020.

MILNER, Hellen. Interests, Institutions, and Information: Domestic Politics and International Relations. New Jersey: Princeton University Press, 1997.

MINISTÉRIO das Relações Exteriores. XI Conferência de chefes de estado e de governo da Comunidade dos Países de Língua Portuguesa - Declaração de Brasília. 01 nov. 2016. Disponível em: <http://www.itamaraty.gov.br/pt-BR/notas-a-imprensa/15107-xiconferencia-de-chefes-de-estado-e-de-governo-da-comunidade-dos-paises-de-linguaportuguesa-declaracao-de-brasilia>. Acessado em 10 de setembro de 2020.

MORAES, Rodrigo Fracalossi de. The parting of the seas: norms, material power and state control over the ocean. Revista Brasileira de Política Internacional, v. 62, n. 1, 2019. MORIN, Jean-Frédéric; ORSINI, Amandine. Essential concepts of global environmental governance. Routledge, 2014.

NATIONS Unies. Résolution adoptée par l'Assemblée générale le 17 novembre 2004, 59/24: Les océans et le droit de la mer. Cinquante-neuvième session, 2004. Disponível em: $<$ https://documents-dds-

ny.un.org/doc/UNDOC/GEN/N04/477/65/PDF/N0447765.pdf?OpenElement>. Acessado em 01 julho de 2019.

. Lettre datée du 30 juin 2011, adressée au Président de l'Assemblée générale par les Coprésidents du Groupe de travail spécial officieux à composition non limitée. Soixante-sixième session, 2011. Disponível em: <https://documents-ddsny.un.org/doc/UNDOC/GEN/N11/397/65/PDF/N1139765.pdf?OpenElement>. Acessado em 
01 julho de 2019.

L'avenir que nous voulons. Rio+20 Conférence des Nations Unies sur le développement durable. 2012. Disponível em: <https://www.diplomatie.gouv.fr/IMG/pdf/declaration_finale_Rio_20_cle0c85b4.pdf>. Acessado em 01 julho de 2019.

Lettre datée du 13 février 2015, adressée au Président de l’Assemblée générale par les Coprésidents du Groupe de travail spécial officieux à composition non limitée. Soixante-neuvième session, 2015a. Disponível em: <https://undocs.org/fr/A/69/780>. Acessado em 01 julho de 2019.

Résolution adoptée par l'Assemblée générale le 19 juin 2015. Soixante-neuvième session, 2015b. Disponível em: <https://undocs.org/fr/A/69/780>. Acessado em 01 julho de 2019.

Document établi par la Présidente pour faciliter les négociations. Conférence intergouvernementale chargée d'élaborer un instrument international juridiquement contraignant se rapportant à la Convention des Nations Unies sur le droit de la mer et portant sur la conservation et l'utilisation durable de la biodiversité marine des zones ne relevant pas de la juridiction nationale, Deuxième session, 2018. Disponível em: <https://undocs.org/fr/A/CONF.232/2019/1>. Acessado em 01 julho de 2019.

Papersmart. [s.l.], [s.d.]. Disponível em: <https://papersmart.unmeetings.org/fr/>. Acessado em 01 julho de 2019.

OECD. Rethinking Innovation for a Sustainable Ocean Economy Report. Directorate for Science, Policy and Innovation. 2019. Disponível em:

<http://www.oecd.org/publications/rethinking-innovation-for-a-sustainable-oceaneconomy-9789264311053-en.htm>. Acessado em 10 de setembro de 2020.

OLIVEIRA, Carina; MALEAN-DUBOIS, Sandrine. Os limites dos termos bem público mundial, patrimônio comum da humanidade e bens comuns para delimitar as obrigações de preservação dos recursos marinhos. Revista de Direito Internacional, v. 12, n. 1, 2015.

ORSINI, Amandine. La construction de la biodiversité en politique internationale à travers l'architecture de son complexe de régimes. In: COMPAGNON, Daniel; RODARY, Estienne. Les Politiques de biodiversité. Paris: Presses de Sciences Po, 2017, p. 27-48.

PERSHING, Andrew J. et al. Oceans and marine resources. In: REIDMILLER, D.R. et al. (orgs.). Impacts, Risks, and Adaptation in the United States: Fourth National Climate Assessment, Volume II. U.S. Global Change Research Program, Washington DC, EUA, p. 353-390. Disponível em: <https://nca2018.globalchange.gov/chapter/9/>. Acessado em 10 de 
setembro de 2020.

PINTO, Paulo Antônio Pereira. A identidade brasileira: Atlântico Sul - "vale a pena ver de novo", por Paulo Antônio Pereira Pinto. Mundorama - Revista de Divulgação Científica em Relações Internacionais. Disponível em: <http://www.mundorama.net/?p=25402>. Acessado em 10 de setembro de 2020.

PUTNAM, Robert. Diplomacy and Domestic Politics: The Logic of Two-Level Games, International Organization, v. 42, n. 3, p. 427-460, 1988.

QUEIROZ, Igor Magri de. The parting of the sea: norms, material power and state control over the ocean? an interview with Rodrigo Fracalossi de Moraes, by Igor Magri. Mundorama, 2019a. Acessado em 10 de setembro de 2020.

QUEIROZ, Igor Magri de. Por que os oceanos? Por uma diplomacia estratégica brasileira para a biodiversidade em alto-mar. Conversas \& Controvérsias, v. 6, n. 1, p. e33358, 2019b.

Disponível em:

$<$ https://revistaseletronicas.pucrs.br/ojs/index.php/conversasecontroversias/article/view/3 3358>. Acessado em 10 de setembro de 2020.

RICARD, Pascale. La conservation de la biodiversité dans les zones maritimes internationales. 2017, 743 f. Tese (Doutorado em Direito Internacional Público) - École doctorale de droit international et européen de la Sorbonne, Université Paris 1 Panthéon Sorbonne, Paris, 2017.

SECRETARIA da Comissão Interministerial para os Recursos do Mar. SECIRM, [s.d.]. Disponível em: <https://www.marinha.mil.br/secirm/>. Acessado em 10 de setembro de 2020.

SILVA, Alexandre Pereira da. Brazil's recent agenda on the sea and the South Atlantic contemporary scenario. Marine Policy, v. 85, p. 25-32, 2017.

TANAKA, Yoshifumi. The international law of the sea. Cambridge University Press, 2015.

TILLER, Rachel et al. The once and future treaty: towards a new regime for biodiversity in areas beyond national jurisdiction. Marine Policy, v. 99, p. 239-242, 2019.

UNCTAD. The Review of Maritime Transport. Genebra: UNCTAD, 2015.

UNEP (United Nations Environment Programme). Global Environmental Outlook, GEO 6. 2019a.

Global Resources Outlook 2019: Natural Resources for the Future We Want. 
Nairobi: A Report of the International Resource Panel, United Nations Environment Programme, 2019b.

VIOLA, Eduardo; BASSO, Larissa. O Sistema internacional no Antropoceno. Revista Brasileira de Ciências Sociais, v. 31, n. 92, Oct. 2016. Disponível em:

<http://www.scielo.br/pdf/rbcsoc/v31n92/0102-6909-rbcsoc-3192012016.pdf>. Acessado em 10 de setembro de 2020 . 